\title{
Postoperative sepsis in infants below 6 months of age
}

\author{
Ulf Kessler, Marc Ebneter, Zacharias Zachariou, Steffen Berger \\ Bern, Switzerland
}

Background: Sepsis is a threatening postoperative complication especially in small infants. Regarding the advances in perinatal medicine, its incidence is unknown to date. We aimed to investigate the incidence, risk factors, laboratory findings and outcome of postoperative sepsis in infants younger than 6 months old.

Methods: We examined postoperative sepsis in babies below 6 months of age during a 4-year period at a tertiary pediatric institution.

Results: The rate of postoperative sepsis was 6.9\%. Laparotomy with enterotomy, thoracotomy and diaphragmatic hernia repair $(P<0.05$, respectively) as well as low postnatal age and long operation time $(P<0.001$, respectively) were correlated with the incidence of sepsis. Significant independent predictors for the development of sepsis were the presence of a central venous catheter and perioperative antibiotic treatment $(P<0.001$, respectively). Coagulase negative Staphylococci were the major infecting organism associated with postoperative sepsis, accounting for $53 \%$ of monomicrobial infections. Complete blood counts with differential were not different between infants with sepsis and controls, who had undergone the same surgical procedures. Outcome was favorable in all cases; however, the length of hospital stay was significantly longer in sepsis patients $(P<\mathbf{0 . 0 5})$.

Conclusions: Postoperative sepsis syndrome is a frequent complication in infants below 6 months of age and causes significant prolongation of hospital stay. Adequate prevention and therapeutic strategies warrant further prospective investigations.

World J Pediatr 2009;5(2):113-117

Author Affiliations: Department of Surgical Pediatrics, Inselspital, Bern University Hospital, and University of Bern, Switzerland (Kessler U, Ebneter M, Zachariou Z, Berger S)

Corresponding Author: Ulf Kessler, MD, Department of Surgical Pediatrics, Inselspital, 3010 Bern, Switzerland and Department of Visceral Surgery, Centre Hospitalier Universitaire Vaudois, 1011 Lausanne, Switzerland (Tel: +41-31-6329223; Fax: +41-31-6329292; Email: ulfkessler@web.de)

doi:10.1007/s12519-009-0022-5

(C)2009, World J Pediatr. All rights reserved.
Key words: central venous catheter; infant; postoperative care; sepsis

\section{Introduction}

7 ostoperative sepsis is a major problem in surgical practice. The morbidity and mortality of postoperative sepsis have been reported to be particularly high in neonates. ${ }^{[1-4]}$ Reports on general pediatric surgery date back to the 1970s and 1980s, but more recent series focus on complications in special fields such as neonatal heart or liver surgery. ${ }^{[5]}$ Regarding the advances made in perinatal medicine, it might be suggested that on the one hand the morbidity and mortality of postoperative sepsis are lower to date. ${ }^{[6]}$ On the other hand, the incidence of postoperative complications in this patient group might also be increasing due to improved survival of conditions formerly causing early death.

Sepsis is defined as a general reaction to a pathogenetic microorganism or its products. ${ }^{[7]}$ Following the 2005 International Consensus Conference on Definitions for Pediatric Sepsis, its diagnosis can be made on the basis of different clinical and laboratory findings with either temperature or leukocyte abnormalities. ${ }^{[7]}$ Ruling out sepsis remains a major challenge to physicians as generalized unspecific reaction can not be clearly differentiated from a specific reaction to an invading microorganism, especially in the postoperative situation in small children.

Routine laboratory parameters such as C-reactive protein (CRP) or differential blood counts are barely helpful since they are regularly altered following surgery. Therefore, in order to avoid postoperative complications, empirical antibiotic therapy is commonly performed. But there is large heterogeneity in the use of perioperative antibiotics. Their application might enhance selection of otherwise less harmful germs and predispose to superinfection. Besides, antibiotic prophylaxis might produce resistance to antibiotics and colonization with gram-negative germs.

The present study was undertaken to retrospectively investigate the incidence, risk factors, laboratory 
findings and outcome of postoperative sepsis in infants below 6 months of age at a single tertiary institution.

\section{Methods}

In 2001 and 2002 we evaluated all charts of operated infants below 6 months of age at the Inselspital, Bern, for morbidity rate and risk factor analysis of postoperative sepsis. For the assessment of laboratory parameters and microbial cultures, we assessed the respective data from all patients with postoperative sepsis from 2001 until 2004. Inclusion criteria were surgery performed in the operation theater under general anesthesia and patient age below 6 months postnatally. Excluded were patients who had undergone diagnostic and therapeutic interventions without skin incision, operations for infectious diseases (necrotizing enterocolitis, intestinal perforation, and abscess evacuation), actual treatment for neonatal sepsis, or more than one surgical procedure within 5 days.

Sepsis was defined according to the criteria of the International Consensus Conference on Definitions for Pediatric Sepsis. ${ }^{[7]}$ Patients were classified as having sepsis when there was systemic inflammatory response syndrome (SIRS) in the presence of or as a result of suspected or proven infections. SIRS was assumed in the presence of at least two of the following four criteria, one of which must be abnormal temperature or leukocyte count: body temperature $338.5^{\circ} \mathrm{C}$ or $<36^{\circ} \mathrm{C}$; tachycardia $>2$ standard deviations (SD) above normal for age in the absence of external stimuli; tachypnea, defined as a mean respiratory rate $>2 \mathrm{SD}$ above normal for age or mechanical ventilation for an acute process not related to neuromuscular disease or receipt of anesthesia; white blood cell count abnormalities ( 0 day to 1 week: $>34 \times 10^{9} / \mathrm{L} ; 1$ week to 1 month: $>19.5$ or $<5$ $\times 10^{9} / \mathrm{L} ; 1$ month to 6 months: $>17.5$ or $<5 \times 10^{9} / \mathrm{L}$; for all ages: $>10 \%$ immature neutrophils). ${ }^{[7]}$

The following parameters were recorded: birth weight (BW), gestational age (GA), gender, Apgar score, mode of delivery (caesarean-section versus vaginal delivery), perinatal complications, duration of preoperative and total hospital stay, type of surgical treatment, length of surgery, presence of a central venous catheter (CVC), perioperative antibiotic prophylaxis and growth of bacterial culture.

Because of the diversity of surgical interventions, the procedures were classified as to their contamination potential (Table 1). In infants with sepsis and in the three procedure groups with the highest risk for sepsis (control group), complete blood cell counts with differential and CRP levels were recorded. In sepsis patients, we recorded laboratory parameters on the day of initial symptoms. In addition, the laboratory parameters taken before and after initial laboratory evaluation for sepsis were recorded. Since the mean duration from operation to symptoms of sepsis was $9 \pm 5$ days, the parameters in the control group were recorded on postoperative day $9 \pm 1$, as well as those taken before and afterwards.

Hematological parameters were evaluated using a Coulter Counter (Celldyn 3500 R, Abbott, IL, USA). Plasma CRP concentrations were quantitated by a latex photometric assay (Hitachi 917, Boehringer Mannheim, Germany).

Statistical analysis was performed using SPSS $^{\odot}$ version 15. Stepwise multivariate logistic regression analysis was used to identify independent factors associated with the occurrence of postoperative sepsis. Associations between variables were determined using Pearson's product-moment correlation coefficient. Differences between the two groups were assessed using Student's $t$ test in case of equivalent variation and normal distribution of values. Otherwise, the MannWhitney $U$ Test was performed. Ordinal data were assessed using the Chi-square test and Fisher's exact test. Changes of laboratory parameters within groups with time were assessed using analysis for repeated measurements. Data were presented as means $\pm \mathrm{SD}$ unless otherwise specified. $P<0.05$ was considered statistically significant.

\section{Results}

A total of 36 infants with postoperative sepsis were included in this study, among them 18 were recruited from 2001-2002 $(6.9 \%, n=260)$, and the other 18 were from 2003-2004.

Postnatal age at surgery was significantly lower and operation time was significantly longer in infants with sepsis as compared to those without $(P<0.001$, respectively). The rate of postoperative use of CVC was significantly higher in infants with sepsis $(72 \%)$ as compared to those without $(8 \%)(P<0.001)$ (Table 2$)$.

Patients undergoing laparotomy with enterotomy, thoracotomy or diaphragmatic hernia repair had a higher risk for postoperative infection with a rate of sepsis of $20 \%, 50 \%$ and $100 \%$, respectively (Table 1 ). However, the three surgical treatments were not independent predictors for the development of postoperative sepsis according to multivariate stepwise regression analysis $(P>0.05)$, despite the presence of a central venous line $(r=2.9, P<0.001)$ and perioperative antibiotic treatment $(r=3.2, P<0.001)$.

There were no differences in the laboratory parameters between patients with sepsis and those 
undergoing comparable procedures (Table 3). In some patients with sepsis, leucocyte count was abnormal.

Table 1. Number of patients per type of surgical procedure with postoperative sepsis during 2001-2002

\begin{tabular}{lcll}
\hline Surgical procedures & $\begin{array}{c}\text { No sepsis } \\
n(\%)\end{array}$ & $\begin{array}{c}\text { Sepsis } \\
n(\%)\end{array}$ & $P$ \\
\hline Hernia repair & $95(100)$ & - & $\mathrm{ns}$ \\
Laparotomy without enterotomy & $12(92)$ & $1(8)$ & $\mathrm{ns}$ \\
Laparotomy with enterotomy & $33(80)$ & $8(20)$ & $<0.05$ \\
Myelocele repair & $6(100)$ & - & $\mathrm{ns}$ \\
Retroperitoneal intervention & $4(100)$ & - & $\mathrm{ns}$ \\
Thoracotomy & $5(50)$ & $5(50)$ & $<0.05$ \\
Urogenital correction & $11(100)$ & - & $\mathrm{ns}$ \\
Plastic surgery with osteotomy & $14(93)$ & $1(7)$ & $\mathrm{ns}$ \\
Orthopedic intervention & $41(98)$ & $1(2)$ & $\mathrm{ns}$ \\
Gastroschisis closure & $1(100)$ & - & $\mathrm{ns}$ \\
Diaphragmatic hernia repair & - & $2(100)$ & $<0.05$ \\
Craniotomy with meningeal incision & $20(100)$ & - & $\mathrm{ns}$ \\
\hline
\end{tabular}

Differences between groups are considered significant at $P<0.05$. ns: not significant.

Table 2. Basic cohort data during the observation period 2001-2002

\begin{tabular}{llll}
\hline Parameters & No sepsis & Sepsis & $P$ \\
\hline Number of patients $(n)$ & 242 & 18 & - \\
Gestational age (wk) & $38.2 \pm 0.2$ & $38.8 \pm 0.7$ & $\mathrm{~ns}$ \\
Birth weight (kg) & $2.98 \pm 0.02$ & $3.02 \pm 0.17$ & $\mathrm{~ns}$ \\
Birth weight percentile (\%) & $42 \pm 2$ & $43 \pm 7$ & $\mathrm{~ns}$ \\
Sex (\% male) & $69 \%$ & $70 \%$ & $\mathrm{~ns}$ \\
Postnatal age (d) & $69 \pm 3$ & $27 \pm 9$ & $<0.001$ \\
Duration of hospital stay (d) & $13 \pm 21$ & $49 \pm 41$ & $<0.001$ \\
Operation time (min) & $82 \pm 4$ & $126 \pm 12$ & $<0.001$ \\
Central venous catheter (\%) & $8 \%$ & $72 \%$ & $<0.001$ \\
\hline
\end{tabular}

Values are mean \pm SD. Differences between groups are considered significant at $P<0.05$. ns: not significant.
But group allocation was not necessarily based on leucocyte counts in any patient since there were other sepsis criteria. ${ }^{[7]}$ No significant changes of laboratory parameters over time could be observed in the two groups. However, patients with sepsis had a trend towards a higher number of immature leucocytes as compared to previous postoperative parameters, follow-up parameters or patients without sepsis $(P>0.05$, respectively).

In 5 patients, culture results were not obtained due to technical reasons. In one patient, cultures remained sterile. In the remaining 30 patients, bacterial growth was positive for blood (9 patients), catheter tip (4), blood and catheter tip (7), catheter tip and pleural effusion (1), catheter tip and tracheobronchial secretion (TBS) (1), TBS (2), pleural effusion (1), TBS and pleural effusion (1), urine (1), peritoneal swab (1), wound swab (1), wound and peritoneal swab (1) (Table 4). Polymicrobial growth was found in 4 cases. Gram-positive bacteria caused $20(77 \%)$ of the monomicrobial infections: 14 isolates (53\%) were coagulase negative Staphylococci (CoNS), $4(15 \%)$ were Staphlococcus aureus, and $2(8 \%)$ were Streptococcus pneumoniae. Gram-negative bacteria caused $6(23 \%)$ of monomicrobial infections: 2 isolates (8\%) were Escherischia coli, 2 (8\%) were Enterococcus species, 1 (4\%) was Enterobacter species, and 1 (4\%) was Serratia liquefaciens.

Outcome was favorable following antibiotic treatment and withdrawal of central lines if present. None of the children with sepsis died during hospital treatment. However, the length of hospital stay was significantly correlated with the occurrence of sepsis as confirmed by regression analysis $(r=3.6, P<0.001)$.

Table 3. Laboratory parameters in patients with sepsis and in controls during 2001-2004

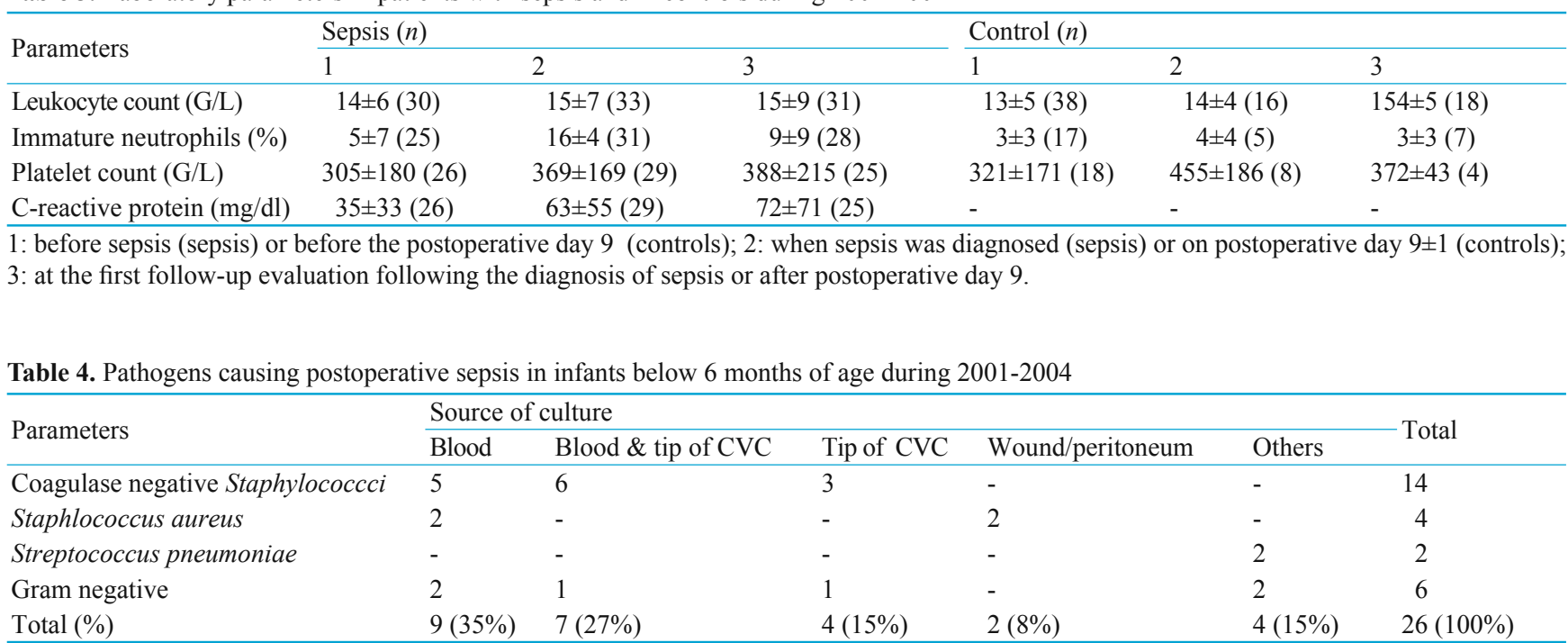

CVC: central venous catheter. 


\section{Discussion}

The first aim of this study was to explore the incidence of postoperative sepsis in infants below 6 months of age. The overall morbidity of postoperative sepsis was about $6.9 \%$ in this study, which is comparable to that in neonates operated on in the 1980s reported by Madden et $\mathrm{al}^{[8]}$ and in infants from birth to 8 years undergoing sternotomy for heart or lung surgery between 1995 and 2003 by Shah et al. ${ }^{[5]}$ In addition, the rate of sepsis for $20 \%$ we found following laparotomy with enterotomy is comparable to that reported during the 1970s and 1980 s. ${ }^{[3]}$ There are few reports of large series on the incidence of postoperative sepsis in infants and newborns. The present study and others are based on single European and North American institution's retrospective investigations and may not fully represent the neonatal populations in the western world. One may nonetheless speculate that the rate of postoperative sepsis has not changed in the past two or three decades. The spectrum of operations however may have changed since more premature and low birth weight infants can undergo surgery nowadays.

The second purpose of this study was to identify risk factors associated with postoperative sepsis. In contrast to the report by Madden el al, ${ }^{[8]}$ we found that perioperative antibiotic prophylaxis does not prevent postoperative infection. Inversely perioperative antibiotic treatment was an independent predictor for sepsis. This phenomenon seems to be paradoxical at the first glance, but Adam and Gross ${ }^{[1]}$ have already described infections and death due to sepsis despite adequate antibiotic prophylaxis and therapy in postoperative neonates. Possible explanations might be on the one hand that the choice to perform antibiotic prophylaxis represents the extent and degree of possible surgical contamination. On the other hand the neonate, especially following surgery, can be considered an immunocompromised host and therefore susceptible to invading microorganisms. Inadequate antibiotic prophylaxis might thus predispose to superinfection with subsequent bacterial invasion. CoNS are usually resistant to ampicillin and variably resistant to amikacin and gentamicin, the most commonly used antibiotics for perioperative prophylaxis in neonates. There is no consensus on optimal antibiotic regimen for neonates or small infants undergoing surgery and practice patterns vary considerably. ${ }^{[9]}$ However, the elevated frequency of postoperative sepsis and the absence of institutional regimens for antibiotic prophylaxis indicate the need of prospective investigations in this field.

Our results confirm that CVCs are a major risk factor and that CoNS are the major infecting organisms associated with postoperative sepsis in infants below 6 months of age. ${ }^{[8,10]}$
In contrast to the previous reports, ${ }^{[1,3]}$ the microorganisms cultured in the present study did not have an enteric predominance. We believe that the correlation between perioperative antibiotic treatment and later sepsis reflects the attitudes of the treating physicians towards a large and possibly non-sterile surgical intervention other than a possible pathophysiological link in between. Furthermore the postoperative conditions of the child, e.g., short bowel, gastroschisis or other situations that do not allow for feeding might also have an impact on the decision whether to insert CVC.

In 6 patients, CoNS were cultured from the catheter tip simultaneously with its culture from blood, and in 8 patients they were from either the CVC tip or blood culture. Besides bacterial growth in blood and catheter tip cultures, bacterial growth from other sources was found frequently. These cultures were all taken simultaneously to the occurrence of SIRS, and isolated bacteria were presumably responsible for sepsis. However, in the 8 patients without a second positive culture, either from blood or from the catheter tip, as well as in the patients with bacterial growth from sites other than blood or catheter tip, we can not definitely distinguish between coincidental sepsis arising from other foci and catheter-related infection. A hindrance in comparability of studies on catheter-related infection is the lack of a unique definition. Chien et $\mathrm{al}^{\left[1{ }^{[1]}\right.}$ required only one positive blood culture, whereas the National Institute of Child Health and Human Development Neonatal Research Network (NICHD) more rigorously required two positive blood cultures. ${ }^{[1]}$ Simultaneous blood cultures drawn through the CVC and peripheral vein, as well as catheter tip cultures in case of removal of the CVC might furthermore help to confirm the diagnosis of CVC related blood stream infection. ${ }^{[12]}$

In the present study, the laboratory data at the onset of symptoms showed no significant changes compared with parameters before disease onset, at septic workup and in the follow-up evaluation. In addition, we found no differences between the septic patients and controls at the time points. We conclude that standard laboratory parameters are barely helpful in clinical decisionmaking. This conclusion is in line with other reports on the usefulness of routine laboratory parameters. ${ }^{[13,14]}$ More modern parameters as procalcitonin, IL-6 or thrombelastography may be helpful in the judgment of a potentially septic infant in the postoperative period, ${ }^{[15,16]}$ but currently no single test fulfills the criteria of an ideal diagnostic test. However, the comparison of laboratory data we made between sepsis and control patients has some important limitations. First, due to the heterogeneity of sepsis patients with large variances in gestational age, postnatal age, 
surgical trauma and others, we did not succeed in defining a perfectly matched control group for the factors possibly influencing the occurrence of sepsis. Second, the retrospective study design is affected by potential sources of bias which may possibly lead to impaired reproducibility of result. In addition, even in a prospective study with narrow inclusion criteria, it is often difficult to clearly differentiate septic from nonseptic patients since there is so far no gold standard for the diagnosis of sepsis. ${ }^{[17]}$

Unlike a high mortality rate of septic infants from the 1970s and 1980s, none of our patients died during hospitalization. ${ }^{[1,3]}$ As reported, we found a prolongation of hospital stay in patients with sepsis. ${ }^{[5,14]}$ The dramatic decrease of mortality might be due to the well-trained professional staff in modern tertiary centers and high awareness of the risk of infection in neonates and small children. But in consideration of the retrospective study design, the reasons for the decrease of mortality and the prolonged hospital stay remain speculative. We assume that the retrospective study design has obvious limitations. In the present study, prophylactic antibiotic therapy was at the discretion of the treating physician and not dictated by a protocol, the antibiotic treatment regimens were not uniform. Therefore, the conclusions on perioperative antibiotic prophylaxis from the present study need to be considered with caution. Taken into consideration the personal and financial consequences of postoperative infections in neonates, the prophylaxis, recognition and treatment of this complication in a potentially increasing population warrants further exploration in multicenter prospective trials.

We conclude that postoperative sepsis syndrome is still a common complication in infants below 6 months of age. Groups of low and high risk can be defined on the basis of the child's age, type and duration of surgery, and the necessity to place a CVC. In contrast to the 1980 s, the mortality following postoperative sepsis is negligible, but the prolongation in the length of hospital stay is significant. Routine laboratory results within normal ranges can not rule out postoperative sepsis. Prospective larger trials may be warranted to develop prophylactic regimens and specific laboratory tools for prevention and early recognition of postoperative sepsis in infancy.

\section{Funding: None.}

Ethical approval: Not needed.

Competing interest: None declared.

Contributors: Kessler U proposed the study and wrote the first draft. Ebneter $\mathrm{M}$ analyzed the data. All authors contributed to the design and interpretation of the study and to further drafts. Berger $\mathrm{S}$ is the guarantor.

\section{References}

1 Adam D, Gross M. Clinical bacteriology of premature and full-term infants dying postoperatively. Prog Pediatr Surg 1979;13:31-38.

2 Hecker WC, Warkotsch A. General survey of the causes of death in patients who died postoperatively in pediatric surgery. Prog Pediatr Surg 1979;13:15-21.

3 Grussner R, Hofmann-von Kap-herr S, Emmrich P. Importance of infection following laparotomy in childhood. Z Kinderchir 1984;39:305-309.

4 Sharma LK, Sharma PK. Postoperative wound infection in a pediatric surgical service. J Pediatr Surg 1986;21:889-891.

5 Shah SS, Kagen J, Lautenbach E, Bilker WB, Matro J, Dominguez TE, et al. Bloodstream infections after median sternotomy at a children's hospital. J Thorac Cardiovasc Surg 2007;133:435-440.

6 Taqi E, Boutros J, Emil S, Dube S, Puligandla P, Flageole H, et al. Evaluation of surgical approaches to pyloromyotomy: a single-center experience. J Pediatr Surg 2007;42:865-868.

7 Goldstein B, Giroir B, Randolph A. International pediatric sepsis consensus conference: definitions for sepsis and organ dysfunction in pediatrics. Pediatr Crit Care Med 2005;6:2-8.

8 Madden NP, Agrawal M, Brereton RJ, Kiely EM, Spitz L. An audit of postoperative sepsis in a neonatal surgical unit. Pediatr Surg Int 1991;6:185.

9 Fallat ME, Mitchell KA. Random practice patterns of surgical antimicrobial prophylaxis in neonates. Pediatr Surg Int 1994;9:479.

10 Chien LY, Macnab Y, Aziz K, Andrews W, McMillan DD, Lee SK. Variations in central venous catheter-related infection risks among Canadian neonatal intensive care units. Pediatr Infect Dis J 2002;21:505-511.

11 Fanaroff AA, Korones SB, Wright LL, Verter J, Poland RL, Bauer $\mathrm{CR}$, et al. Incidence, presenting features, risk factors and significance of late onset septicemia in very low birth weight infants. The National Institute of Child Health and Human Development Neonatal Research Network. Pediatr Infect Dis J 1998;17:593-598.

12 Raad I, Hanna H, Maki D. Intravascular catheterrelated infections: advances in diagnosis, prevention, and management. Lancet Infect Dis 2007;7:645-657.

13 Kessler U, Mungnirandr A, Nelle M, Nimmo AF, Zachariou Z, Berger S. A simple presurgical necrotizing enterocolitismortality scoring system. J Perinatol 2006;26:764-768.

14 Klein MD, Rood K, Graham P. Central venous catheter sepsis in surgical newborns. Pediatr Surg Int 2003;19:529-532.

15 Kessler U, Guenther P, Zachariou Z. The relationship between coagulation and the extend of surgery and postoperative infection in surgical infants below 6 months of age. Clin Hemorheol Microcirc 2006;34:447-452.

16 Chiesa C, Pellegrini G, Panero A, Osborn JF, Signore F, Assumma $\mathrm{M}$, et al. C-reactive protein, interleukin-6, and procalcitonin in the immediate postnatal period: influence of illness severity, risk status, antenatal and perinatal complications, and infection. Clin Chem 2003;49:60-68.

17 Fischer JE, Harbarth S, Agthe AG, Benn A, Ringer SA, Goldmann DA, et al. Quantifying uncertainty: physicians' estimates of infection in critically ill neonates and children. Clin Infect Dis 2004;38:1383-1390.

Received May 16, 2008

Accepted after revision November 17, 2008 